\title{
Next-Generation Sequencing Analysis Identified Genomic Alterations in Pathological Morphologies of 3 Cases of Pulmonary Carcinosarcoma
}

This article was published in the following Dove Press journal: OncoTargets and Therapy

\section{Fan $\mathrm{Li}$ \\ Shan $\mathrm{Hu}$ \\ Kangle Kong \\ Peng Cao \\ Peng Han (iD) \\ Yu Deng \\ Bo Zhao}

Department of Thoracic Surgery, Tongji Hospital of Tongji Medical College, Huazhong University of Science and Technology, Wuhan 430030, People's Republic of China
Correspondence: Bo Zhao Email13006369600@I63.com
Background: Pulmonary carcinosarcomas (PCSs) are a heterogeneous group of non-smallcell lung carcinomas (NSCLCs) with aggressiveness and a poor prognosis. Although genetic mutations of some common lung cancer subtypes have been extensively studied, the molecular characteristics of PCSs and the existence of abnormal target genes are unknown.

Methods: In this study, the clinical and molecular characterization in 3 pulmonary sarcomatoid carcinomas (PSCs) were presented using microscope analysis and next-generation sequencing (NGS) analysis.

Results: The results revealed a carcinosarcomas subtype presenting squamous cell carcinoma and sarcoma components in all 3 cases. NGS analysis showed that 182, 316 and 230 shared mutations were detected between sarcoma and lung carcinoma from 3 patients. Two identical alterations in two genes (CSMD3 and RYR3) that were all shared by the two components in 3 patients. Tumor suppressor gene TP53 $(5 / 6,83 \%)$ showed the highest mutation frequency for driver genes here. Additionally, we focused on an LYST mutation which was mainly present in the sarcoma components. Moreover, the clonal evolution and signature analysis confirm that lung squamous cell carcinoma and sarcoma in each PCS patient may have come from a common ancestor, and mutagenesis was possibly related to indirect effects of tobacco, age or other unknown factors.

Conclusion: Our results indicate that genetic analysis and molecular targeted therapy are necessary for the identification and treatment of these rare lung tumors. CSMD3 and LYST, as common mutation genes, may be a potential therapeutic target in PCS.

Keywords: non-small cell lung cancer, pulmonary carcinosarcoma, lung squamous cell carcinoma, sarcoma, genetic analysis

\section{Introduction}

Pulmonary carcinosarcoma (PCS) is a rare lung malignancy, which is classified as a subtype of the pulmonary sarcomatoid carcinoma (PSC). ${ }^{1}$ And its sarcoma components have heterogenous differentiation, representing only $0.1 \%$ to $0.3 \%$ in all lung malignancies. ${ }^{2,3}$ Similar to other types of lung cancer, PSC tends to affect older patients with a history of smoking and is mainly male. ${ }^{4,5}$ However, as with other treatments for non-small cell lung cancer, even early PSC has a poor prognosis. In the past decade, with the widespread use of next-generation sequencing (NGS), the treatment of lung cancer (mainly adenocarcinoma) has made substantial progress, because some molecular abnormalities have been identified and proved to be successful biomarkers and/or drug targets (eg, anaplastic lymphoma kinase 
$(A L K)$ rearrangement and epidermal growth factor receptor (EGFR) mutations). ${ }^{6,7}$ Although genetic mutations in some common lung cancer subtypes have been extensively studied, the molecular characteristics of PSC and the presence of abnormal target genes are still unknown. Existing chemotherapy for PSC is highly ineffective, so targeted therapy is a very attractive treatment option to improve outcomes. $^{4,8}$ And it is imperative to search for diagnostic or prognostic biomarkers and identify potential therapeutic targets for PSC. The purpose of this study was to investigate the potential target gene abnormalities in 3 pulmonary carcinosarcomas previously diagnosed by morphology and immunohistochemistry.

\section{Materials and Methods}

Three patients diagnosed with primary pulmonary carcinosarcoma who underwent surgery at Tongji hospital affiliated to Huazhong University of Science and Technology between May 2018 and June 2019 were enrolled in this study. All subjects provided informed consent, and the study protocol was approved by the clinical research ethical review board at Tongji hospital affiliated to Huazhong University of Science and Technology. The diagnosis was based on the morphology of (hematoxylin-eosin) HE staining, and the tumor cell content greater than 50\% was determined by two experienced pathologists. Immunohistochemical (IHC) analysis was subsequently performed including biomarkers such as cytokeratin (CK), cytokeratin 7 (CK7), carcinoembryonic antigen (CEA), and epithelial membrane antigen (EMA), etc. The squamous and sarcoma components of lung carcinosarcoma were microscopically divided. The DNA was extracted separately using the FFPE Plus LEV DNA Purification Kit (Promega, Madison, WI). DNA quality and quantity were assessed using a Nanodrop and Qubit (Thermo Fisher Scientific, Waltham, MA). Sequencing libraries of different components were prepared using the KAPA Hyper Prep kit (KAPA Biosystems) with an optimised manufacturer's protocol. Enriched libraries were amplified and detected on Illumina Hiseq4000 NGS platform (Illumina) in accordance with the manufacturer's instructions. WES sequencing was performed to detect somatic mutations. The mean coverage depth of WES was approximately 130x for controls and 200x for tumors samples. The sequencing data in the FASTQ format were mapped to the human genome (hg19) using BWA aligner 0.7.10. Local alignment optimization, variant calling and annotation were performed using GATK 3.2, MuTect, and VarScan, respectively.

\section{Results}

\section{Clinical Features of the Study Cohort}

The clinical data of the 3 cases are summarized in Table 1. They had smoked for an average of 30 years. Three patients were all men, with mean age of 63 years (range, 55-71). Per current World Health Organization criteria, cases were all composed of carcinosarcoma. Their tumors averaged about 6 centimeters in size. Two patients presented with tumors in the right lobe, and 1 patient presented with a tumor in the left lobe. In the pathologic staging, 2 patients had clinical Stage III A disease, 1 patient had Stage II B disease. Three patients all accepted surgical treatment with Lobectomy and systematic mediastinal lymphadenectomy, respectively. Only the stage II B patients also received chemotherapy. At last followup, they were all alive.

\section{Pathological Findings}

According to the World Health Organization, there are five histological subtypes of PSC: pleomorphic carcinoma, spindle cell carcinoma, giant cell carcinoma, carcinosarcoma, and pulmonary blastoma. Compared with the other three subtypes, carcinosarcoma and pulmonary blastoma are relatively rare. In our study, three patients were all diagnosed with pulmonary carcinosarcoma, as shown in Figure 1. Pathological findings of case1 (P1) showed moderate to poorly differentiated squamous cell carcinoma with myxoid lesions in sarcoma components. Case 2 (P2) is a moderately differentiated squamous cell carcinoma with pleomorphic sarcoma, in which sarcoma is accompanied by bone and cartilage differentiation. Similarly, the case 3 (P3) showed moderate to poorly differentiated squamous cell carcinoma with sarcoma component as undifferentiated sarcoma conformation.

The immunohistochemical results of 3 patients are shown in Table 2. All 3 cases (100\%) stained positive for $\mathrm{CK} 5 / 6$ and $\mathrm{P} 63$ and were reactive to $\mathrm{Ki67}$ (70\%, $30 \%$ and $60 \%$, respectively). Cases 2 and 3 were both reactive to EMA, VIM (vimentin) and CK8/18, and all cases were negative for Syn (synaptophysin) and CgA (chromogranin A).

\section{Genetic Findings}

The NGS results showed that most of the variant classification in the three patients were missense mutation (Figure 2A), and the most these mutations were single nucleotide polymorphisms (SNP, 95\%), followed by deletions (DELs, 4\%) or 
Table I Clinical Features of the 3 Patients with Pulmonary Sarcomatoid Carcinoma

\begin{tabular}{|c|c|c|c|c|c|c|c|c|c|c|c|}
\hline Patients & Sex & Age & $\begin{array}{l}\text { Adjuvant } \\
\text { CT }\end{array}$ & Surgery & $\begin{array}{l}\text { Smoking } \\
\text { History }\end{array}$ & $\begin{array}{l}\text { Tumor } \\
\text { Location }\end{array}$ & Subtypes & pTNM & Stage & Chemotherapy & Survival \\
\hline $\mathrm{PI}$ & Male & 55 & Yes & Pneumonectomy & $20 / d$ & RLL & CS & T3NOMO & $\| B$ & Yes & Yes \\
\hline P2 & Male & 62 & Yes & Pneumonectomy & $40 / d$ & RMLL & CS & T4NOMO & IIIA & No & Yes \\
\hline P3 & Male & 71 & Yes & Lobectomy & $30 / d$ & LLL & CS & T4NOMO & IIIA & No & Yes \\
\hline
\end{tabular}

Abbreviations: P, patient; LLL, left lower lobe; RMLL, right middle and lower lobe; RML, right lower lobe; d, day; CS, carcinosarcoma.

insertions, as shown in Figure 2B. Most patients showed C>T-predominant mutation profiles (39\%) (Figure 2C). For case1, of the 293 total putative somatic variants, 13 variants were considered driver gene mutations. ${ }^{9}$ Fifteen of 445 putative somatic variants were driver gene mutations for case 2 , and 29 of 675 in case3. The three patients had eight common mutated genes including MUC17, LYST, SLIT2, EIF4G1,
MUC16, CSMD3, RYR3, and driver gene TP53. CSMD3 and $R Y R 3$ were found in both lung squamous cell carcinoma and sarcoma tissue of each case, followed in frequency by MUC17, SLIT2 and TP53 (5/6, 83\% of tissue samples) (Figure 2D). The most frequent driver alterations involved the following genes: $\operatorname{TP} 53(83 \%, 5 / 6)$, KIT $(67 \%, 4 / 6)$, $A P O B(67 \%, 4 / 6)$ and $C D K N 2 A(67 \%, 4 / 6)$ (Figure 2E). The
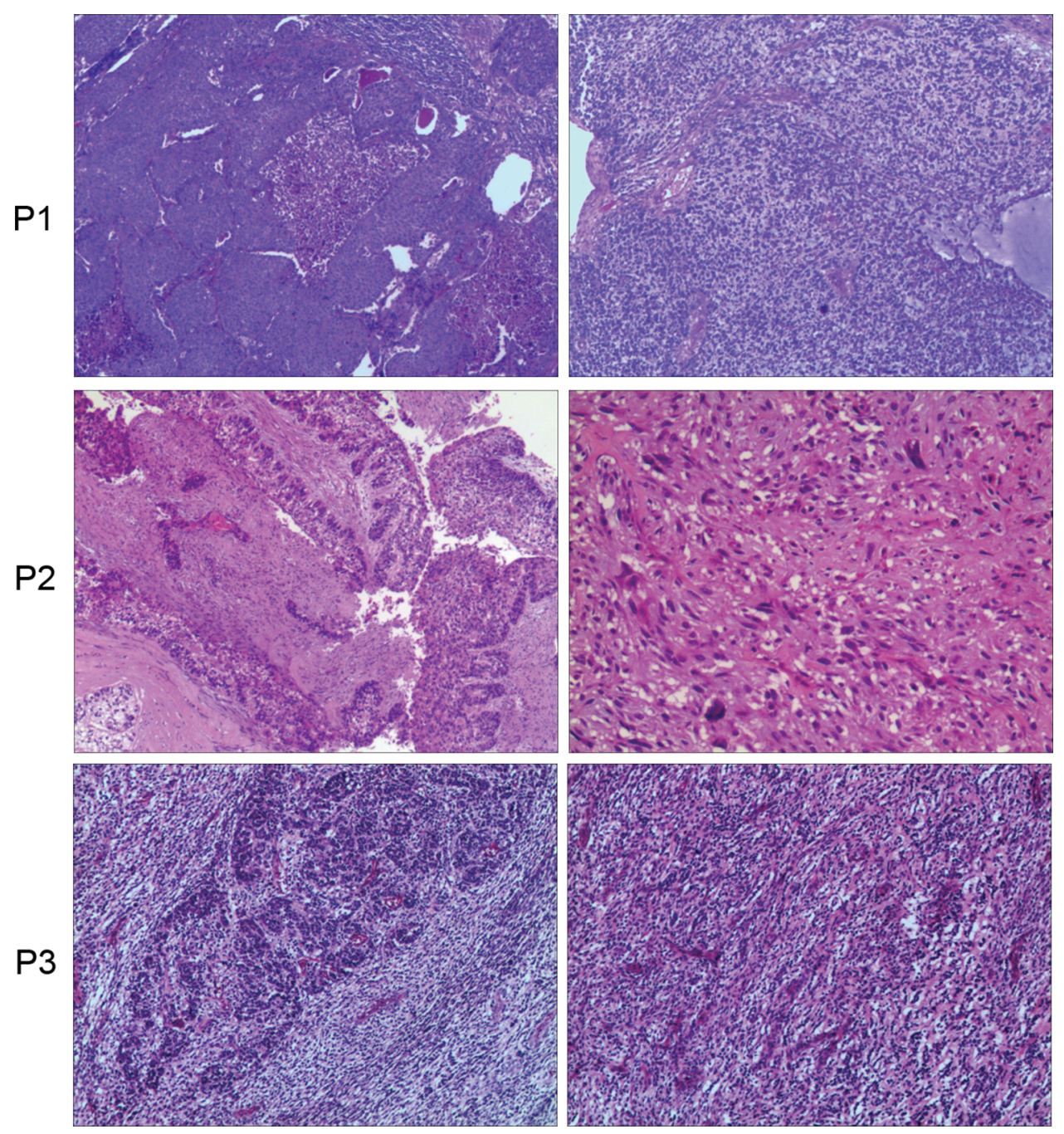

Figure I Pathological characteristics demonstrated by H\&E staining. Case ( $\mathrm{PI}$ ) showed moderate to poorly differentiated squamous cell carcinoma with myxoid lesions in sarcoma components. Case 2 (P2) is a moderately differentiated squamous cell carcinoma with pleomorphic sarcoma, in which sarcoma is accompanied by bone and cartilage differentiation. Case 3 (P3) showed moderate to poorly differentiated squamous cell carcinoma with sarcoma component as undifferentiated sarcoma conformation. 
Table 2 Immunohistochemical Results for the 3 Patients with Pulmonary Sarcomatoid Carcinoma

\begin{tabular}{|l|l|}
\hline \multicolumn{2}{|l|}{ Immunohistochemistry } \\
\hline Patient I & \\
CK5/6, P63, P40, CD56, P53, Ki67 (70\%) & Positive \\
TTFI, NapsinA, CK7, ALK(D5F3), C-MET, ROSI, CgA, & Negative \\
Syn & \\
\hline Patient 2 & \\
PCK, EMA, CK8/18, CK5/6, P63, P40, P53, c-MET, & Positive \\
VIM, MDM2, SATB2, CDK4, PI6, FLII, MyoDI, CD56, & \\
Ki67 (30\%) & \\
ALK(D5F3), ROSI, CD34, SMA, Desmin, Caldesmon, & Negative \\
S-I00, SOXI0, Myogenin, CD3I, ERG, Syn, CgA & \\
\hline Patient 3 & \\
PCK, EMA, CK8/18, CKI9, CK5/6, P63, P40, VIM, & Positive \\
CD68, Ki67 (60\%), MCM2 (40\%) & \\
CK7, TIFI, NapsinA, ROSI, C-MET, ALK(D5F3), P53, & Negative \\
STAT6, SMA, Desmin, Caldesmon, MyoDI, CD3I, & \\
Myogenin, CD34, ERG, FLII, S-I00, CD56, Syn, CgA, & \\
\hline
\end{tabular}

Note: Bold font: the common tumor markers for pulmonary and sarcoma tissue. Abbreviations: CK, cytokeratin; CK7, cytokeratin 7; EMA, epithelial membrane antigen; CK5/6, cytokeratin 5/6; TTF-I, thyroid transcription factor-I; CgA, chromogranin A; Syn, synaptophysin.

variant classification in different tissues of the same patient are basically similar, but not in different patients.

Each patient's lung squamous cell carcinoma tissue and sarcoma tissue have both private and shared mutation genes shown in Figure 3. 182, 316 and 230 shared mutations were detected between different tumors from patients 1, 2 and 3 (Figure 3A). Two common mutation genes CSMD3 and RYR 3 were shared in 3 patients and 6 tissues (Figure 3B). Heatmap of validated mutations showed that 15,12 and 5 shared mutations were detected, respectively, between patient 1 and 2, patient 2 and 3 and patient 1 and 3 (Figure 3C). For the driver gene mutations (Figure 3D-F), 9, 14, and 14 shared mutations were detected between different tumors from each patient. But no driver gene was shared in 6 tissues. Of them, 3 gene mutations were shared in the sarcoma components, and LYST mutation was specific (Figure 4A and B). We found LYST-missense mutations only in the sarcoma components of 2/3 cases, but LYST-nonsense mutation occurred in both lung squamous cell carcinoma and sarcoma components in another patient.

\section{Clonal Evolution and Signatures}

In addition, we performed clonal evolution analysis to examine the heterogeneity of tumor evolution in different tumors of each patient, respectively. Phylogenetic tree analysis based on squamous cell carcinoma and sarcoma showed that they had different evolutionary processes (Figure 5A-C). Clones of different lesions follow their own evolutionary path to form different branches. There were 8-9 clusters in each case, and the number of single nucleotide variants (SNVs) in the trunk varied from 6 to 680. There are gene mutations were shared in these subclones between the spatially distinct tumors in any two patients, but not in all three patients (Figure 5D). These results were consistent with histopathological results, indicating that the lung squamous cell carcinoma and sarcoma in each patient may have come from a common ancestor.

In order to determine the relationship between mutation frequency distribution of pulmonary carcinosarcoma samples and cosmic signature, non-negative matrix decomposition was carried out on the frequency of 96 substitution types in all samples (Figure 6A). And signatures 1, 4, 6 and 24 were identified in all patients (Figure 6B). These signatures were likely to be due to age (signature 1) tobacco-induced mutations (signature 4) and defective DNA mismatch repair (signature 6). There is not much difference in signature analysis between the lung squamous cell carcinoma tissue and sarcoma tissue of each patient, which further supports our above results.

\section{Discussion}

Pulmonary sarcomatoid carcinoma (SC) is an aggressive type of lung cancer with poor prognosis and with strong inter- and intra-tumor heterogeneity. ${ }^{10,11}$ Pulmonary carcinosarcoma, one of the five subtypes, is relatively rare and has been little studied. Compared with women, carcinosarcoma occurs mainly in male smokers, and the average age of patients is 65 years. ${ }^{12}$ And the frequency of epithelial components is as follows: squamous cell carcinoma (46\%), adenocarcinoma (31\%), and adenosquamous carcinoma (19\%). ${ }^{12}$ Sarcoma components include rhabdomyosarcoma, chondrosarcoma, osteosarcoma, and combinations of these components. ${ }^{13}$ In our study, all three male smokers were diagnosed with pulmonary carcinosarcoma, presenting as squamous cell carcinoma with sarcoma component. For immunohistochemistry, EMA, CK5/6, CK7, TIF1, P63, chromogranin A, CD56, and synaptophysin can be used as markers for carcinomatous components, and desmin, vimentin, and smooth muscle/sarcomeric actin can be used as markers for sarcomatous elements. ${ }^{12,14}$

The mutational characteristics of pulmonary carcinosarcoma are difficult to determine because it is relatively 


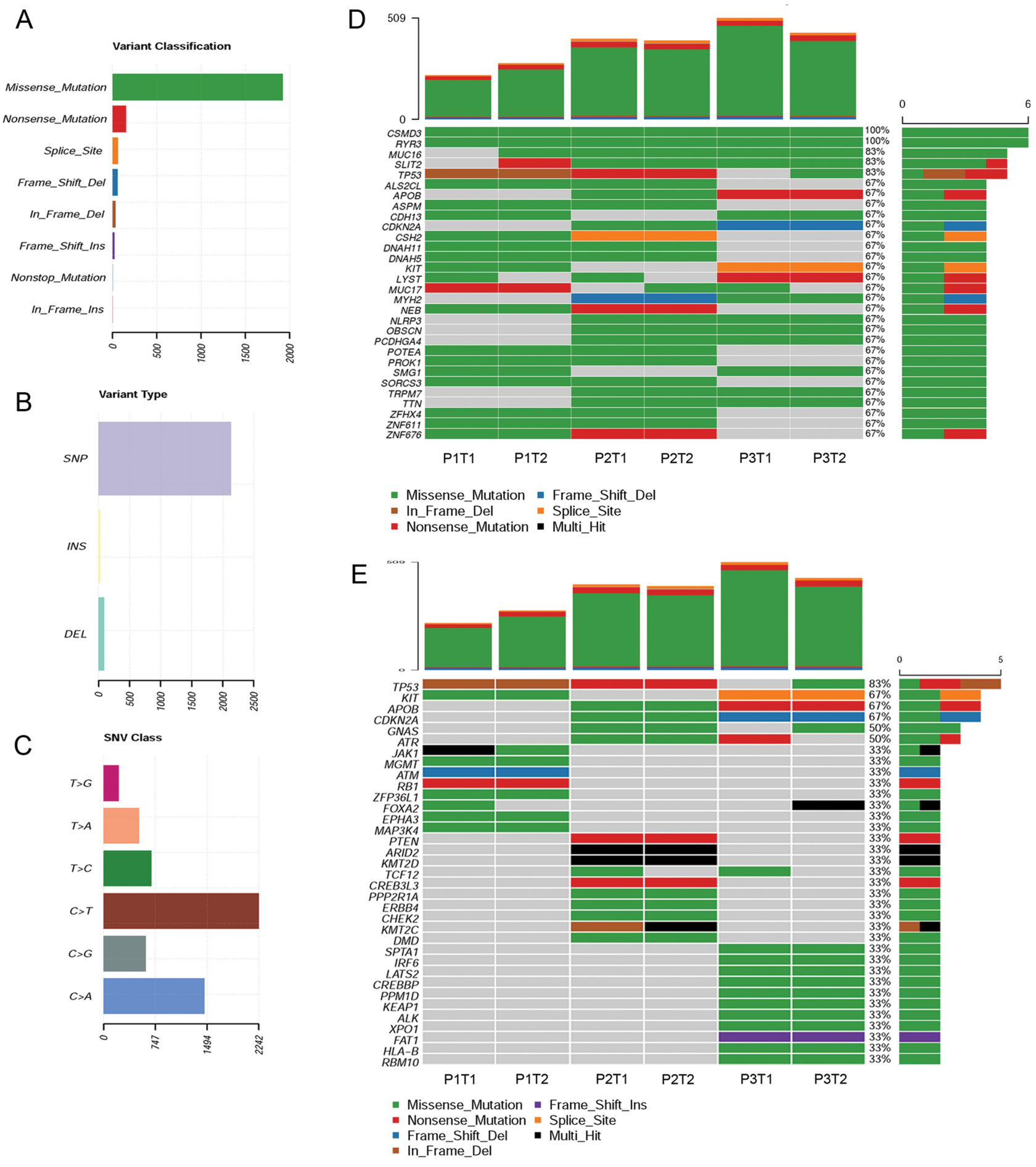

Figure 2 The genetic mutational landscape and patterns of 3 PCS patients including lung squamous cell carcinoma and sarcoma tissues. The variant classification (A), variant types (B) and SNV class (C)of 6 samples. We classified the somatic mutations (D) and driver gene mutations (E) for each sample. The different colored tables represent different of mutations. Abbreviations: Tumor I, sarcoma component; Tumor 2, lung squamous cell carcinoma.

rare compared to other more common histological types. However, molecular analysis of PCS is an important step to better understand the molecular basis and potential treatment options for this aggressive tumor. Here, we identified intertumor molecular variants at different sites of PCS and discovered several mutation profiles for PSCs Each patient's lung squamous cell carcinoma tissue and sarcoma tissue have both private and shared mutation genes. TP53, as known as tumor suppressor, showed the high mutation frequency here. Study had indicated that the 

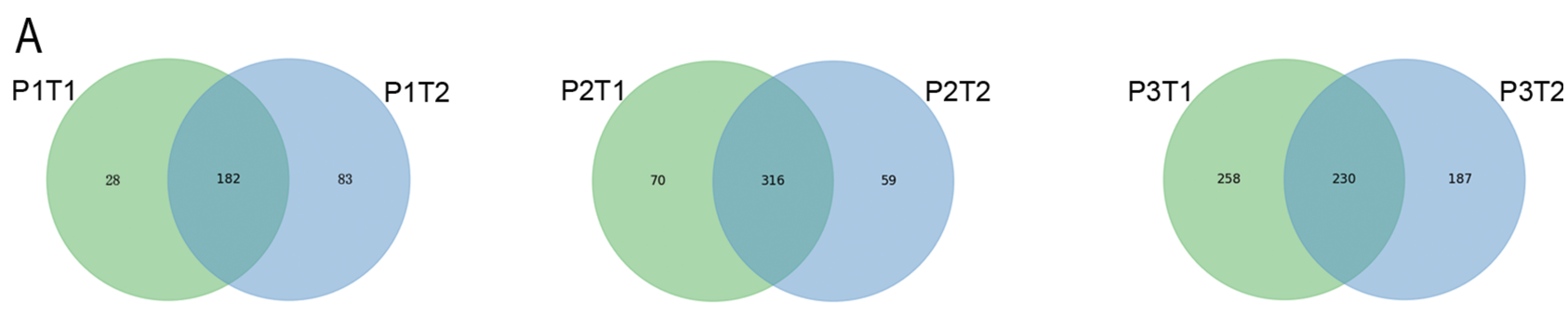

B

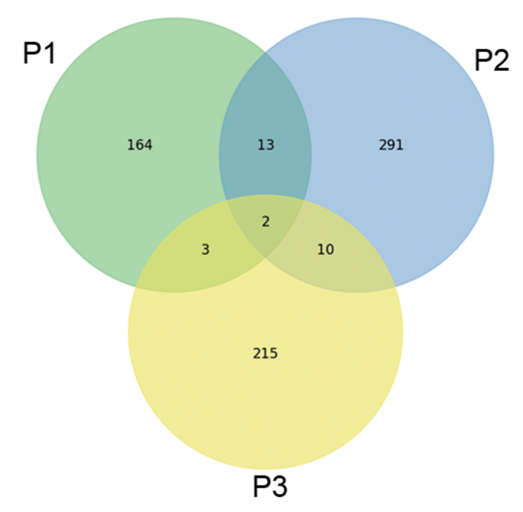

D

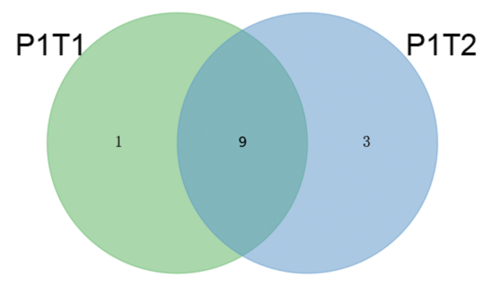

$\mathrm{E}$

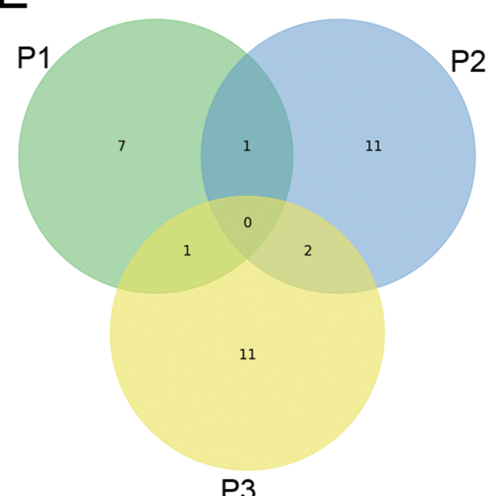

C

\begin{tabular}{l|c|c|c|c|c|c|}
\cline { 2 - 7 } P1T1 & 210 & 182 & 15 & 15 & 5 & 5 \\
\cline { 2 - 7 } P1T2 & 182 & 265 & 15 & 15 & 5 & 5 \\
P2T1 & 15 & 15 & 386 & 316 & 12 & 12 \\
\cline { 2 - 7 } P2T2 & 15 & 15 & 316 & 375 & 12 & 12 \\
\cline { 2 - 7 } P3T1 & 5 & 5 & 12 & 12 & 488 & 230 \\
\cline { 2 - 7 } P3T2 & 5 & 5 & 12 & 12 & 230 & 417 \\
\cline { 2 - 7 } & P1T1 P1T2 P2T1 P2T2 P3T1 P3T2
\end{tabular}
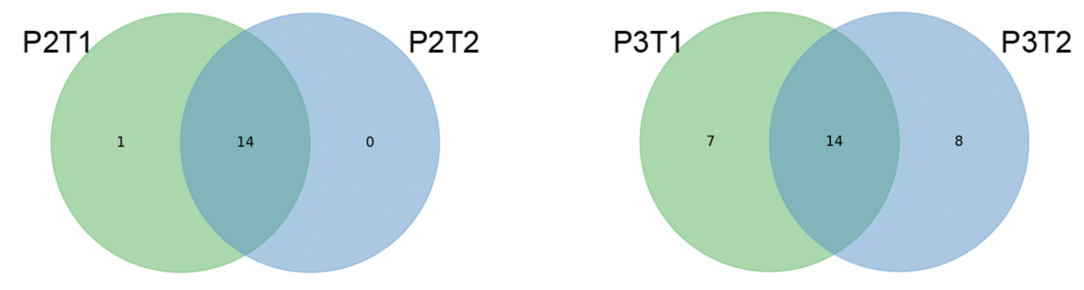

$\mathrm{F}$

\begin{tabular}{l|c|c|c|c|c|c|}
\cline { 2 - 7 } P1T1 & 10 & 9 & 1 & 1 & 1 & 1 \\
\cline { 2 - 7 } P1T2 & 9 & 12 & 1 & 1 & 1 & 1 \\
\cline { 2 - 7 } P2T1 & 1 & 1 & 15 & 14 & 2 & 2 \\
\cline { 2 - 7 } P2T2 & 1 & 1 & 14 & 14 & 2 & 2 \\
\cline { 2 - 7 } P3T1 & 1 & 1 & 2 & 2 & 21 & 14 \\
\cline { 2 - 7 } P3T2 & 1 & 1 & 2 & 2 & 14 & 22 \\
\cline { 2 - 7 } & P1T1 P1T2 P2T1 P2T2 P3T1 P3T2
\end{tabular}

Figure 3 Similarity among different lesions rising from a single patient with PCSs based on somatic mutation analysis. (A) Venn diagram illustrating the distributions of genetic mutation in lung squamous cell carcinoma and sarcoma tissues of each patient. (B) Venn diagram illustrating the distributions of genetic mutation in 3 patients. (C) Heatmap of validated mutations shared by the 3 PCS patients with 6 tissues. (D) Venn diagram illustrating the distributions of driver genetic mutation in lung squamous cell carcinoma and sarcoma tissues of each patient. (E) Venn diagram illustrating the distributions of driver genetic mutation in 3 patients. (F) Heatmap of validated driver gene mutations shared by the 3 PCS patients with 6 tissues.

Abbreviations: Tumor I, sarcoma component; Tumor 2, lung squamous cell carcinoma. 

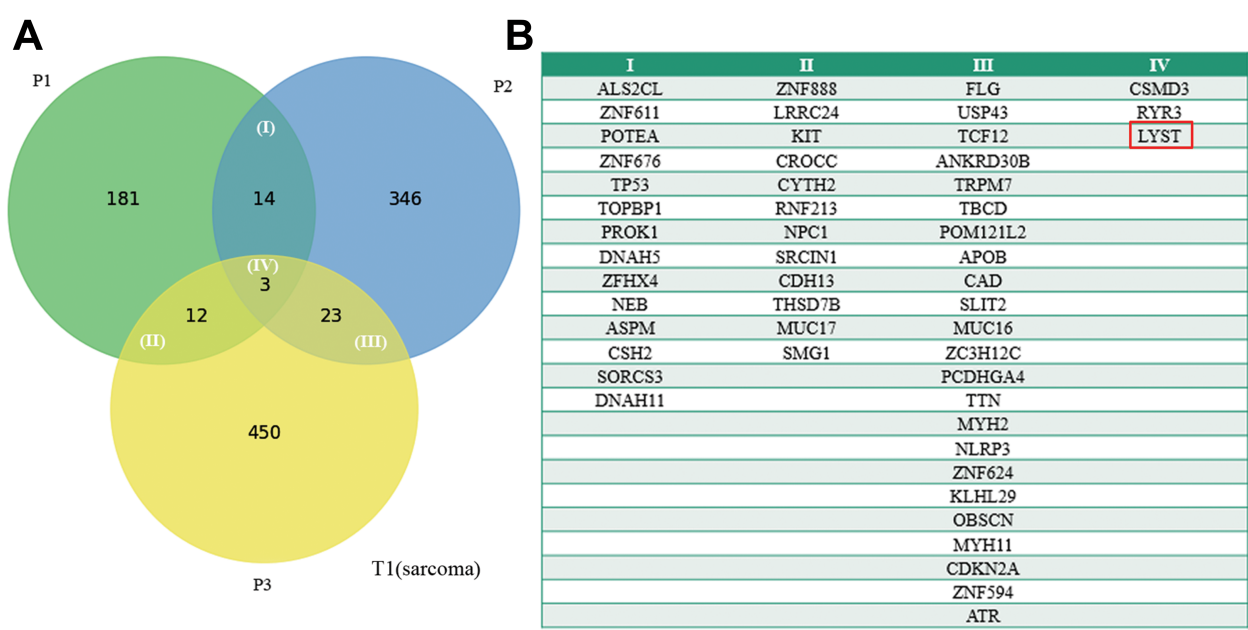

Figure 4 Mutations in sarcoma component. (A) A Venn diagram depicting the overlap of the mutated genes. (B) The names of the genes in each category of the Venn diagram.

A
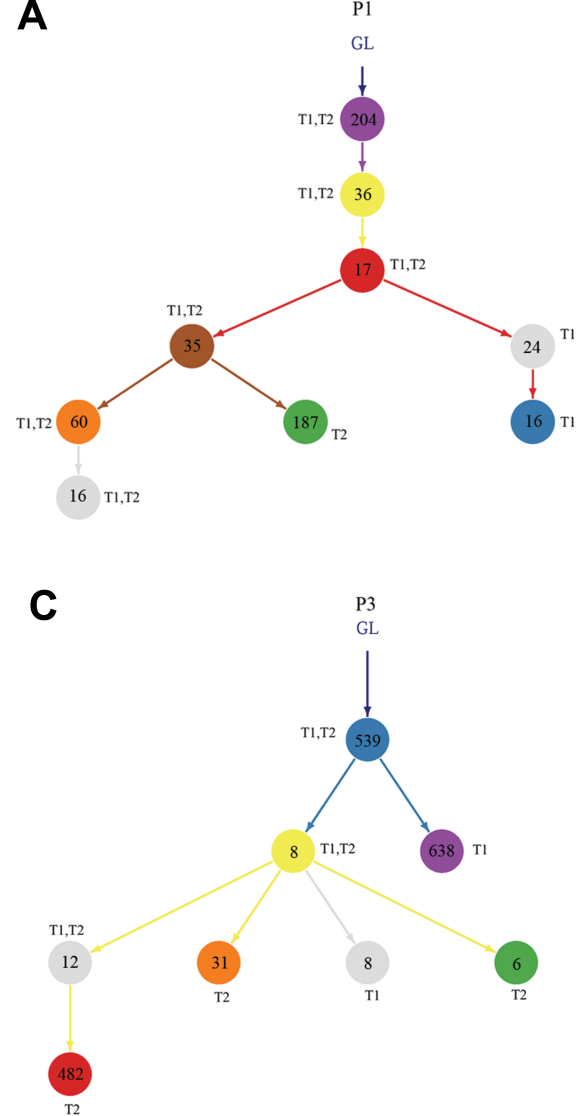

B

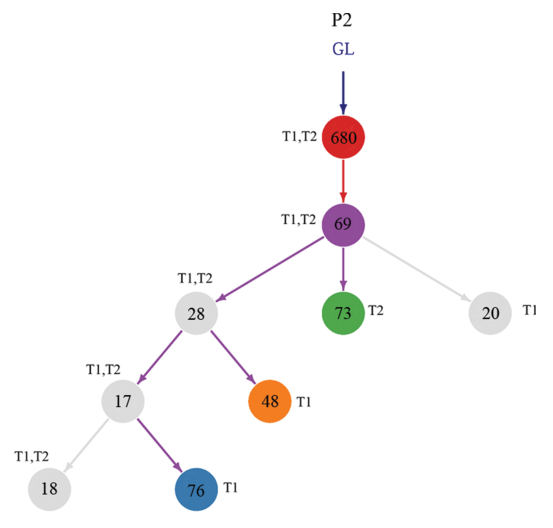

D

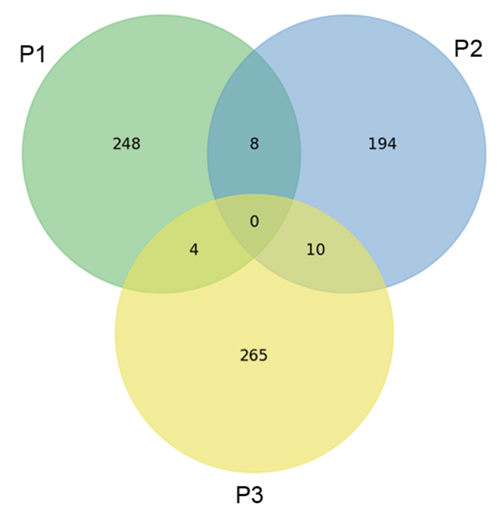

Figure 5 Intratumor spatial heterogeneity with squamous cell carcinoma and sarcoma tissue in 3 PCS patients. Phylogenetic tree plotted by revolver showing the clonal evolution of each patient. Patient I (A), Patient 2 (B) and Patient 3 (C). The grey circle means the cluster without driver gene mutations, and the color circle means the cluster with one or more driver gene mutations. Numbers in the circle represent numbers of SNVs in each cluster. GL, germline. (D) Venn diagram illustrating the distributions of gene mutations in these subclones for 3 PCS patients.

Abbreviations: Tumor I, sarcoma component; Tumor 2, lung squamous cell carcinoma.

incidence of TP53 point mutations in adenocarcinoma is approximately $27 \%$, squamous cell carcinoma is $43 \%$ and polymorphic cancer is $14 \% .{ }^{15}$ Another study showed that mutations in the TP53 gene occurred in $55 \%$ of PSC cases. ${ }^{16}$ In our study, all three patients had a TP53 mutation, but the variant classification was different in different 
A

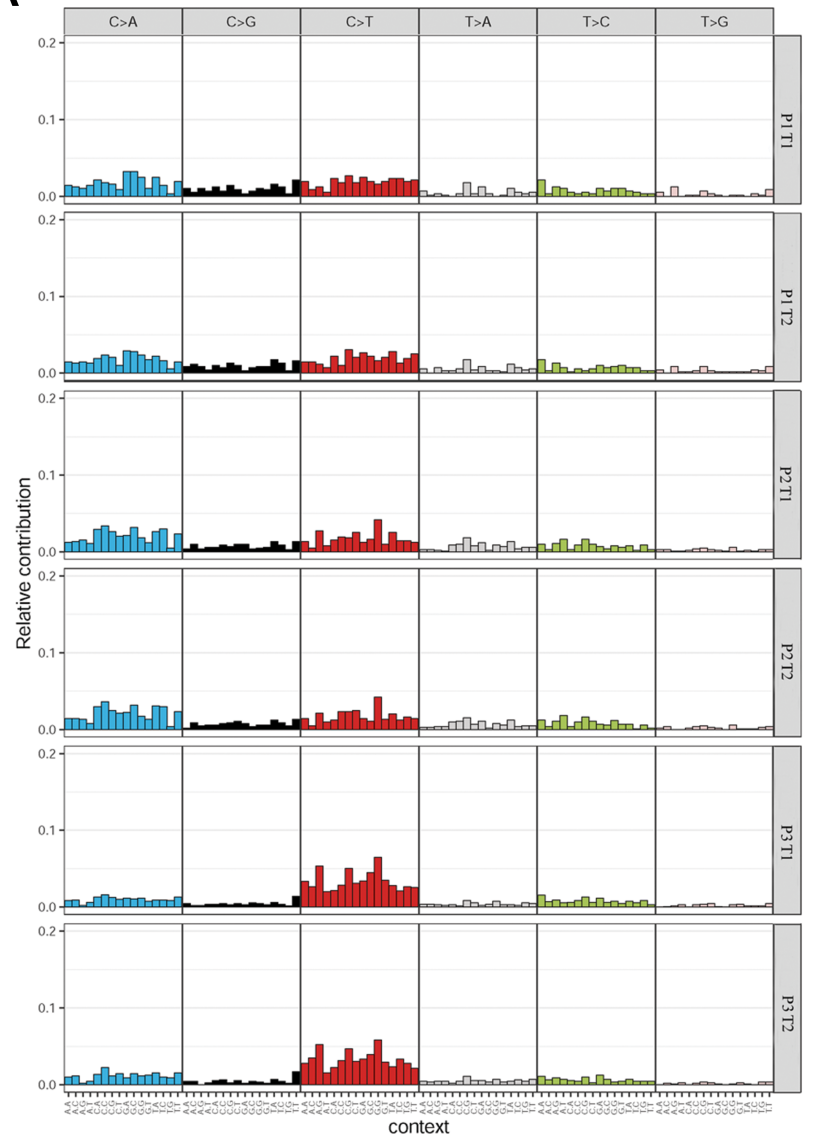

B

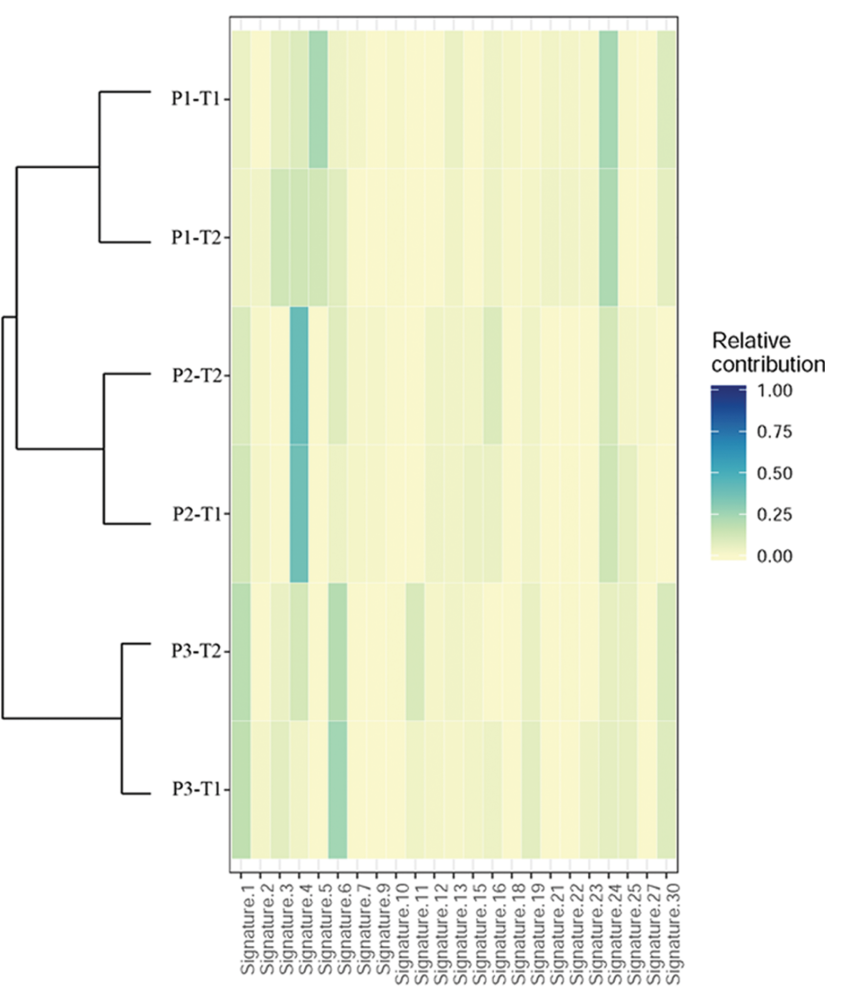

Figure 6 Mutational signature analyses of 3 PCS patients. Non-negative matrix decomposition was carried out on the frequency of 96 substitution types in all samples (A); Mutation frequency distribution of pulmonary carcinosarcoma samples of cosmic signature (B).

Abbreviations: Tumor I, sarcoma component; Tumor 2, lung squamous cell carcinoma.

patients, including In-Frame-Del, Nonsense mutation and Missense mutation. Two common mutation genes CSMD3 and RYR3 were shared in all 3 patients and 6 tissues. Qiu et al found that CSMD3 and RYR1 can accurately predict sensitivity to etoposide in small-cell lung cancer. ${ }^{17}$ And the efficacy of chemotherapy for PSCs is unsatisfactory, which was associated with significant side effects in all NSCLC patients, including PSC, because of the lack of specificity of chemotherapy. ${ }^{18}$ Moreover, in lung squamous cell carcinoma, CSMD3 mutations were significantly associated with patient prognosis, ${ }^{19}$ which suggests that CSMD3 may be a potential therapeutic target. Interestingly, an LYST mutation was mainly present in the sarcoma component of 3 patients, indicating that it may be a sarcoma-specific mutation. LYST-missense mutation was present in 2 of the 3 patients and LYST-nonsense substitution was present in the other 1 patient. The enrichment of LYST mutations in sarcoma may be an accidental observation, or it may be the result of active selection that drives mutation. LYST gene mutations are associated with Chediak-Higashi syndrome (CHS), a rare autosomal recessive lysosomal disease with blood and immune abnormalities. $^{20}$ In addition to CHS, mutations in the LYST gene are one of the key factors leading to lifethreatening diseases characterized by hemophagocytic lymphohistiocytosis, defective immune system function, and uncontrolled activation of $\mathrm{T}$ cells and macrophages. ${ }^{21}$ Tarpey et al found that LYST may represent a novel cancer gene through loss of function mutations in chordoma. ${ }^{22}$ Therefore, can we boldly speculate that LYST may be a specific alteration in the formation of sarcoma, although it still needs to be verified by a large number of samples and tests.

In addition, we performed clonal evolution analysis to examine the heterogeneity of tumor evolution in different tumors of each patient, respectively. There are lots of gene mutations were shared in these subclones between the lung squamous cell carcinoma and sarcoma in each patient, 
indicating that the lung squamous cell carcinoma and sarcoma in each patient may have come from a common ancestor although we are aware that our conclusions are limited by the study's small sample size. In order to determine the relationship between mutation frequency distribution of pulmonary carcinosarcoma samples and cosmic signature, non-negative matrix decomposition was carried out on the frequency of 96 substitution types in all samples. All our patients were current or past smokers, the tobacco-related signature 4 was the predominant signature in these cases. Tobacco-related feature 4 is mainly composed of $\mathrm{C}>\mathrm{A} / \mathrm{G}>\mathrm{T}$ translocation, which may be due to the guanine adduct of benzo [A] pyrene, a component of tobacco smoke, ${ }^{23}$ suggesting that smoking directly affects the occurrence of signature 4 tumors. This hypothesis was confirmed by $\mathrm{C}>\mathrm{A} / \mathrm{G}>\mathrm{T}$ transcriptional asymmetry and a greater proportion of signature 4 in clone mutations compared to subcloning. Moreover, signatures 1, 6 and 24 were identified in all tissues. Signature 1 was likely to be due to age and signature 6 may be due to defective DNA mismatch repair. PCS had unique clinical features compared to common types of NSCLCs in terms of lymphatic invasion and surgical treatment. Elderly patients may be associated with a poorer prognosis. ${ }^{24}$ And there was little difference in the signature analysis of the lung squamous cell carcinoma and sarcoma tissue for each patient, which further supported our findings.

\section{Conclusion}

Although our sample size was small, due to sample time constraints, our genetic analysis confirms that lung squamous cell carcinoma and sarcoma in each PCS patient may have come from a common ancestor. Mutagenesis was possibly related to indirect effects of tobacco, age or other unknown factors. Furthermore, TP53 gene has a higher mutation frequency in PCS which is similar to some previous studies, suggesting that PCS is a distinct subtype of NSCLC. CSMD3 and LYST, as common mutation genes, may be a potential therapeutic target due to its gene function and high mutation rate in PCS.

\section{Ethics and Consent Statement}

This study was approved by the Ethics Committee of Tongji hospital affiliated to Huazhong University of Science and Technology. All patients provided written informed consent. Informed consent was obtained from each subject in accordance with the Declaration of Helsinki.

\section{Funding}

This work was supported by the Hubei Natural Science and Technology Fund Youth Fund (No. CFB295). The authors thank Shanghai Tongshu Biotechnology Co., Ltd. for technical support.

\section{Disclosure}

The authors declare that they have no competing interests for this work.

\section{References}

1. Travis WD. Pathology of pulmonary vasculitis. Semin Respir Crit Care Med. 2004;25(5):475-482. doi:10.1055/s-2004-836141

2. Chang YL, Lee YC, Shih JY, Wu CT. Pulmonary pleomorphic (spindle) cell carcinoma: peculiar clinicopathologic manifestations different from ordinary non-small cell carcinoma. Lung Cancer. 2001;34(1):91-97. doi:10.1016/S0169-5002(01)00224-0

3. Travis WD, Travis LB, Devesa SS. Lung cancer. Cancer. 1995;75(1 Suppl):191-202.

4. Huang SY, Shen SJ, Li XY. Pulmonary sarcomatoid carcinoma: a clinicopathologic study and prognostic analysis of 51 cases. World J Surg Oncol. 2013;11252.

5. Lin Y, Yang H, Cai Q, et al. Characteristics and prognostic analysis of 69 patients with pulmonary sarcomatoid carcinoma. Am J Clin Oncol. 2016;39(3):215-222. doi:10.1097/COC.0000000000000101

6. Li T, Kung HJ, Mack PC, Gandara DR. Genotyping and genomic profiling of non-small-cell lung cancer: implications for current and future therapies. J Clin Oncol. 2013;31(8):1039-1049. doi:10.1200/ JCO.2012.45.3753

7. Devarakonda S, Morgensztern D, Govindan R. Genomic alterations in lung adenocarcinoma. Lancet Oncol. 2015;16(7):e342-351. doi:10.1016/S1470-2045(15)00077-7

8. Ouziane I, Boutayeb S, Mrabti H, et al. Sarcomatoid carcinoma of the lung: a model of resistance of chemotherapy. N Am J Med Sci. 2014;6 (7):342-345. doi:10.4103/1947-2714.136920

9. Bailey MH, Tokheim C, Porta-Pardo E, et al. Comprehensive characterization of cancer driver genes and mutations. Cell. 2018;174 (4):1034-1035. doi:10.1016/j.cell.2018.07.034

10. Nakajima M, Kasai $T$, Hashimoto $H$, Iwata $Y$, Manabe $H$. Sarcomatoid carcinoma of the lung: a clinicopathologic study of 37 cases. Cancer. 1999;86(4):608-616. doi:10.1002/(SICI)1097-0142(19990815)86:4<608::AID-CNCR9>3.0.CO;2-1

11. Martin LW, Correa AM, Ordonez NG, et al. Sarcomatoid carcinoma of the lung: a predictor of poor prognosis. Ann Thorac Surg. 2007;84 (3):973-980. doi:10.1016/j.athoracsur.2007.03.099

12. Koss MN, Hochholzer L, Frommelt RA. Carcinosarcomas of the lung: a clinicopathologic study of 66 patients. Am J Surg Pathol. 1999;23(12):1514-1526. doi:10.1097/00000478-199912000-00009

13. Kotlowska MP, Rueda AG, Olmedo ME, et al. Efficacy of immunotherapy in sarcomatoid lung cancer, a case report and literature review. Respir Med Case Rep. 2019;26310-26314.

14. Franks TJ, Galvin JR. Sarcomatoid carcinoma of the lung: histologic criteria and common lesions in the differential diagnosis. Arch Pathol Lab Med. 2010;134(1):49-54.

15. Mochizuki T, Ishii G, Nagai K, et al. Pleomorphic carcinoma of the lung: clinicopathologic characteristics of 70 cases. Am J Surg Pathol. 2008;32(11):1727-1735. doi:10.1097/PAS.0b013e3181804302

16. Lococo F, Gandolfi G, Rossi G, et al. Deep sequencing analysis reveals that kras mutation is a marker of poor prognosis in patients with pulmonary sarcomatoid carcinoma. J Thorac Oncol. 2016;11 (8):1282-1292. doi:10.1016/j.jtho.2016.04.020 
17. Qiu Z, Lin A, Li K, et al. A novel mutation panel for predicting etoposide resistance in small-cell lung cancer. Drug Des Devel Ther. 2019;132021-132041.

18. Vieira T, Girard N, Ung M, et al. Efficacy of first-line chemotherapy in patients with advanced lung sarcomatoid carcinoma. $J$ Thorac Oncol. 2013;8(12):1574-1577. doi:10.1097/01.JTO.0000437008.00554.90

19. La Fleur L, Falk-Sorqvist E, Smeds P, et al. Mutation patterns in a population-based non-small cell lung cancer cohort and prognostic impact of concomitant mutations in KRAS and TP53 or STK11. Lung Cancer. 2019;13050-13058.

20. Al-Tamemi S, Al-Zadjali S, Al-Ghafri F, Dennison D. Chediakhigashi syndrome: novel mutation of the CHS1/LYST gene in 3 Omani patients. J Pediatr Hematol Oncol. 2014;36(4):e248-250. doi:10.1097/MPH.0000000000000025

21. Jessen B, Maul-Pavicic A, Ufheil $\mathrm{H}$, et al. Subtle differences in CTL cytotoxicity determine susceptibility to hemophagocytic lymphohistiocytosis in mice and humans with chediak-higashi syndrome. Blood. 2011;118(17):4620-4629. doi:10.1182/blood2011-05-356113
22. Tarpey PS, Behjati S, Young MD, et al. The driver landscape of sporadic chordoma. Nat Commun. 2017;8(1):890. doi:10.1038/ s41467-017-01026-0

23. Nik-Zainal S, Kucab JE, Morganella S, et al. The genome as a record of environmental exposure. Mutagenesis. 2015;30(6):763-770. doi:10.1093/mutage/gev073

24. Sun L, Dai J, Wang X, et al. Pulmonary carcinosarcoma: analysis from the surveillance, epidemiology and end results database. Interact Cardiovasc Thorac Surg. 2020;30(1):4-10. doi:10.1093/ icvts/ivz215

\section{Publish your work in this journal}

OncoTargets and Therapy is an international, peer-reviewed, open access journal focusing on the pathological basis of all cancers, potential targets for therapy and treatment protocols employed to improve the management of cancer patients. The journal also focuses on the impact of management programs and new therapeutic agents and protocols on patient perspectives such as quality of life, adherence and satisfaction. The manuscript management system is completely online and includes a very quick and fair peer-review system, which is all easy to use. Visit http://www.dovepress.com/ testimonials.php to read real quotes from published authors. 